Meta

Journal des traducteurs

Translators' Journal

\title{
Bibliographie sélective pour la traduction des sciences biomédicales
}

\section{Sylvie Vandaele}

Volume 46, numéro 1, mars 2001

Traduction médicale et documentation / Medical translation and documentation

URI : https://id.erudit.org/iderudit/004535ar

DOI : https://doi.org/10.7202/004535ar

Aller au sommaire du numéro

Éditeur(s)

Les Presses de l'Université de Montréal

ISSN

0026-0452 (imprimé)

1492-1421 (numérique)

Découvrir la revue

Citer ce document

Vandaele, S. (2001). Bibliographie sélective pour la traduction des sciences

biomédicales. Meta, 46(1), 117-127. https://doi.org/10.7202/004535ar d'utilisation que vous pouvez consulter en ligne.

https://apropos.erudit.org/fr/usagers/politique-dutilisation/ 


\title{
Bibliographie sélective pour la traduction des sciences biomédicales
}

\author{
SYLVIE VANDAELE \\ Université de Montréal, Montréal, Canada
}

Pourquoi parler de sciences biomédicales plutôt que de médecine? Parce que les frontières entre la médecine et les sciences connexes, ces dernières qualifiées, il n'y a pas si longtemps, de "fondamentales", sont de plus en plus floues. En témoignent, par exemple, les nouveaux modes de fabrication de médicaments qui font appel à des techniques de biologie moléculaire ou à des organismes modifiés génétiquement. Le traducteur médical se verra donc inévitablement placé devant des textes qui, s'ils traitent essentiellement de clinique, de pharmacologie ou de pharmacie, évoquent des notions de biologie cellulaire ou moléculaire, de biochimie ou de génétique.

Dans la sélection bibliographique que nous avons réalisée, nécessairement subjective et incomplète, nous avons tenté de réunir un ensemble d'ouvrages fondamentaux qui devraient permettre au traducteur de se repérer dans le labyrinthe notionnel et terminologique de la biomédecine moderne. En ce qui concerne les sous-domaines de la médecine, nous nous sommes limités à ceux qui sont actuellement "à la mode ", tels que la cardiologie, l'immunologie, les neurosciences, etc. car, à moins d'entreprendre un travail d'envergure dans une spécialité donnée, le traducteur trouvera le plus souvent les renseignements qui lui seront nécessaires dans les manuels de référence généraux ou encyclopédiques. Sauf cas particulier, nous avons retenu les ouvrages les plus récents. Dans chaque section, nous proposons des ouvrages en français et en anglais, que nous avons classés par ordre chronologique inverse.

On s'étonnera peut-être de trouver la traduction française de certains ouvrages, essentiellement américains. Dans les cas retenus, un tel choix nous semble justifié: il s'agit d'ouvrages pratiquement "incontournables » et les traductions ont été réalisées par ou avec des équipes de spécialistes renommés. En fait, on ne peut plus, comme par le passé, affirmer que les textes rédigés par des auteurs francophones sont plus fiables que les textes traduits. Il se trouve que c'est parfois l'inverse (et c'est tout à l'honneur des équipes de traduction): l'influence de l'anglais chez les rédacteurs francophones, obligés de publier leurs articles de recherche presque exclusivement en anglais et peu au fait des pièges de transfert linguistique, est parfois bien plus sensible que chez les traducteurs... S'il arrive que l'usage finisse par entériner des termes criticables, il reste toutefois une marge de manœuvre appréciable, en particulier pour les néologismes, puisque les traductions, en tant que textes participant à l'usage d'un domaine, peuvent donner l'occasion de privilégier certaines solutions préférables à d'autres. Dans tous les cas, on ne saurait trop recommander la plus grande vigilance et un esprit critique acéré, quel que soit le document consulté, tout comme il est indispensable de confronter plusieurs sources, quelle que soit la langue.

Il ne nous a bien entendu pas été possible de consulter ni de mentionner tous les ouvrages sur le marché. Par conséquent, le fait qu'un livre n'apparaisse pas dans la présente bibliographie ne signifie en aucune façon qu'il soit à rejeter a priori. Enfin, 
l'astérisque $\left(^{*}\right)$ indique les volumes qui devraient constituer les outils de base du traducteur médical.

\section{Dictionnaires}

*Delamare, J. et al. (2000): Le Garnier-Delamare. Dictionnaire des termes de médecine, 26" éd., Paris, Maloine, $991 \mathrm{p}$.

*PoLonovski, J. (2000): Dictionnaire de biologie, Paris, Conseil international de la langue française, $967 \mathrm{p}$.

Bonaff́, I-I (2000): Distinnnaire de dermatologie pédiatrique, $2^{\complement}$ éd., Paris, Maloine, $424 \mathrm{p}$.

Civatte, J. (2000): Dictionnaire de dermatologie, Paris, Conseil international de la langue française, $516 \mathrm{p}$.

Delamare, J. (2000) : Dictionnaire Maloine de l'infirmière, $2^{\mathrm{e}}$ éd., Paris, Maloine, $552 \mathrm{p}$.

FARCoT, C. (2000): Petit lexique d'orthopédie anglais/français, Montpellier, Sauramps, 240 p.

*Manuila, L. et al. (1999): Dictionnaire médical, 8 e éd., Paris, Masson, 704 p.

Auffray, C. et A. JACQuard (1998): Le petit Trésor: dictionnaire de la biologie, Paris, Flammarion, $317 \mathrm{p}$.

BERESNIAK, A. et al. (1998): Dictionaire raisonné des termes des entreprises du médicament, Paris, Flammarion Médecine Sciences, vii $+195 \mathrm{p}$.

${ }^{*}$ Kernanum, S. et al. (1998): Dictionnaire de médecine Flammarion, 6"éd., Paris, Flammarion, $1064 \mathrm{p}$.

Buican, D. (1997): Dictionnaire de biologie: notions essentielles, Paris, Larousse, 166 p.

Delaveau, P., dir. (1997): Dictionnaire des sciences pharmaceutiques et biologiques, Paris, Louis Pariente, 3 vol.

Auray, J.-P. et al. (1996): Dictionnaire commenté d'économie de la santé, Paris, Masson, xiii + $289 \mathrm{p}$.

BAINS, W. et al. (1996): Dictionnaire des biotechnologies, Paris, Association française de normalisation (AFNOR), $450 \mathrm{p}$. [trad. de Biotechnology from $A$ to $Z$ ].

*Gladstone, W. J. (1996): Dictionnaire anglais-français des sciences médicales et paramédicales, $4^{\mathrm{e}}$ éd., Saint-Hyacinthe, Edisem; Paris, Maloine, 1282 p.

Maugras, M. (1996): Dictionnaire de biologie cellulaire, Paris, Ellipses, 174 p.

DUPAYRAT, J. (1996): Dictionnaire chimique et technologique des sciences biologiques / Chemical and Technological Dictionary of Biological Sciences, $2^{\circ}$ éd., Londres, Techniques et documentations, $229 \mathrm{p}$.

Caignon, P. et al. (1995): Dictionnaire anglais-français et français-anglais des biotechnologies, Saint-Hyacinthe, Edisem, 185 p.

BESNIER, J.-P. (1995) : Dictionnaire d'anatomie locomotrice, Paris, Arthos, 584 p.

NAKos, D. (1994): Dictionnaire de l'imagerie médicale / Dictionary of Medical Imaging, Sainte-Foy, Presses de l'Université Laval; Paris, Masson, $x+94 \mathrm{p}$.

*Jammal, A. et al. (1988): Dictionnaire d'épidémiologie, Saint-Hyacinthe, Edisem; Paris, Maloine, $171 \mathrm{p}$.

*Andreoli, T. E. et al., ed. (2000): Dorland's Illustrated Medical Dictionary, 29th ed., Philadelphia, Saunders, xxiii +2085 p.

${ }^{*}$ SMith, A. D., ed. (2000): Oxford Dictionary of Biochemistry and Molecular Biology, Oxford, Oxford University Press, $x i+738 \mathrm{p}$.

${ }^{*}$ DAY, S. (1999): Dictionary of Clinical Trials, Chichester, Wiley, $x+217$ p.

LACKIE, J. M. and J. A. T. Dow (1999): The Dictionary of Cell \& Molecular Biology, 3rd ed., San Diego, London and New York, Academic Press, 500 p.

Pugr, M. B. et al., ed. (1999): Stedman's Medical Dictionary, 27th ed., Baltimore, Lippincott Williams \& Wilkins, xxxvi $+2098 \mathrm{p}$.

Anderson, K. N. et al., ed. (1998): Mosby's Medical Dictionary, 5th ed, St. Louis, Mosby, 1752 p.

Taliaferro-Blauvelt, C. and R. T. F. Nelson (1998): A Manual of Orthopaedic Terminology, 6th ed., Saint Louis, Mosby, xii +463 p. 
Thомаs, C. L., ed. (1997): Taber's Cyclopedic Medical Dictionary, 18th ed., Philadelphia, F. A. Davis Co., xxiv + 2439 p.

MeInert, C. (1996): Clinical Trials Dictionary: Terminology and Usage Recommendations, Baltimore, Johns Hopkins Center for Clinical Trials, cviii +363 p.

Rognehaugh, R. (1996): The Managed Health Care Dictionary, Gaithersburg, Aspen Publishers, xiii +211 p.

Cruse, J. M. and R. E. Lewis (1995): Illustrated Dictionary of Immunology, Boca Raton, CRC Press, 330 p.

Last, J. L., ed. (1995): A Dictionary of Epidemiology, 3rd ed., New York and Toronto, Oxford University Press, xvii $+180 \mathrm{p}$.

Segen, J. C. (1995): Current Medtalk, Stamford, Appleton \& Lange, 984 p.

Winslade, J. and D. R. Hutchinson (1992): Dictionary of Clinical Research, Brookwood, Brookwood Medical Publications, 137 p.

\section{Terminologie médicale et étymologie}

Bossy, J. (1999): La grande aventure du terme médical, Montpellier, Sauramps Médical, 487 p.

${ }^{*}$ Quérin, S. (1998): Dictionnaire des difficultés du français médical, Saint-Hyacinthe, Edisem, xi + $200 \mathrm{p}$.

Godrie, A. et T. Hervieux (1997): Terminologie médicale et physiopathologie, Paris, Nathan Technique, $256 \mathrm{p}$.

Sournia, J.-C. (1997) : Langage médical français, Toulouse, Privat; Paris, Editions de Santé, 157 p.

Bossy, J. (1997): Etymologie et structures des termes médicaux, Montpellier, Sauramps Médical, $229 \mathrm{p}$.

${ }^{*}$ Cheval.lier, J. (1995): Précis de terminologie médicale: introduction au domaine et au langage médical, $6^{e}$ éd., Paris, Maloine, 315 p.

Delaveau, P. (1995): La mémoire des mots en médecine, pharmacie et sciences, Paris, Louis Pariente, $382 \mathrm{p}$.

${ }^{*}$ Lespérance, B. (s. d.): Passe-partout, petit lexique des racines grecques et latines, Montréal, Entreprises Normand Michel Inc.

Birmingham, J. J. (1999): Medical Terminology, a Self-Learning Text, 3rd ed., Saint Louis, Mosby, $\mathrm{xv}+570 \mathrm{p}$. [avec cédérom].

Gylys, B. A. and M. E. Wedding (1999): Medical Terminology. A System Approach, 4th ed., Philadelphia, F.A. Davis Co., 398 p.

Stanaszek, W. F. et al. (1998): Understanding Medical Terms. A Guide for Pharmacy Practice, 2nd ed., Lancaster and Basel, Technomic Publishing Co., xii +424 p.

Fremgen, B. F. (1997): Medical Terminology: An Anatomy and Physiology Systems Approach, Upper Saddle River, Brady Prentice Hall, xxiii +440 p.

SCHWager, E. (1991): Medical English Usage and Abusage, Phoenix, Oryx Press, xiv +216 p.

\section{Rédaction médicale}

Huguier, M. et al. (1998) : La rédaction médicale. De la thèse à l'article original. La communication orale, Paris, Doin, 165 p.

JeNICEK, M. (1997): Casuistique médicale: bien présenter un cas clinique, Saint-Hyacinthe, Edisem, $\mathrm{xix}+113 \mathrm{p}$.

Bénichoux, R. (1997): Guide de la communication médicale et scientifique: comment écrire, comment dire (en français et en anglais), Montpellier, Sauramps, 214 p.

Huth, E. J. (1999): Writing and Publishing in Medicine, 3rd ed., Baltimore, Williams \& Wilkins, $\mathrm{x}+348 \mathrm{p}$.

JENICEK, M. (1999): Clinical Case Reporting in Evidence-Based Medicine, Oxford, ButterworthHeinemann, $\mathrm{xx}+154 \mathrm{p}$.

Iverson, C. et al., ed. (1998): American Medical Association Manual of Style: A Guide for Authors and Editors, 9th ed., Baltimore, Williams \& Wilkins, xi +660 p. 


\section{Traduction médicale}

Rouleau, M. (1994): La traduction médicale. Une approche méthodique, Brossard, Linguatech, $326 \mathrm{p}$.

VAN Hoof, H. (1986): Précis pratique de traduction médicale (anglais-français), Paris, Maloine, $309 \mathrm{p}$.

\section{Ouvrages de référence}

\subsection{Encyclopédies et ouvrages généraux}

*Naudin, C. et N. Grumbach, dir. (2000): Larousse médical, Paris, Larousse, xvi + 1203 p.

${ }^{*}$ Fattorusso, V. et O. Ritter (1998): Vademecum clinique: du diagnostic au traitement, 15 éd., Paris, Masson, $1785 \mathrm{p}$.

*Berkow, R. et A. J. Fletcher, dir. (1994): Manuel Merck de diagnostic et thérapeutique, $2^{e}$ éd., Paris, Éditions d'Après, xxx +2767 p. [trad. de la $16^{\mathrm{e}}$ éd. américaine].

HAmburger, J. et M. Leporrier (1993): La petite encyclopédie médicale Hamburger: guide de pratique médicale, $18^{e}$ éd., Paris, Flammarion Médecine Sciences, xiv +1747 p.

Encyclopédie médico-chirurgicale (1971-), Paris, Éditions Techniques [collection à révision périodique].

Akos. Encyclopédie pratique de médecine (1998), Paris, Elsevier [collection à révision périodique].

*BeERs, M. H. and R. Berkow, eds. (1999): The Merck Manual of Diagnosis and Therapy, 17th ed., Rahway (N. J.), Merck Research Laboratories, xxxiii +2833 p. [offert sur cédérom ou en ligne sur Internet : <http://www.merck.com/pubs/mmanual >)

\subsection{Biologie cellulaire et biologie moléculaire}

Étienne-DeCANt, J. et al. (1999) : Biochimie génétique, biologie moléculaire, $5^{\mathrm{c}}$ éd., Paris, Masson, $\mathrm{xx}+505 \mathrm{p}$.

AlBerts, B. et al. (1999): L'essentiel de la biologie moléculaire. Introduction à la biologie moléculaire de la cellule, trad. fr. Serge Perleman, Paris, Flammarion Médecine Sciences, xxii +630 p. + $\mathrm{G} 18+\mathrm{R} 62+\mathrm{I} 30$.

Alliet, J. (1997): Cytobiologie, Paris, Ellipses, 860 p.

PoIrier, J. et al. (1997): Histologie moléculaire, $5^{\text {e }}$ éd., Paris, Masson, 432 p.

Lodish, H. et al., dir. (1997): Biologie moléculaire de la cellule, Bruxelles, De Boeck, XLvII + 1344 p. [trad. de la $3^{\mathrm{e}}$ éd. de Molecular Cell Biology].

Alberts, B. et al. (1995): Biologie moléculaire de la cellule, $3^{e}$ éd., trad. fr. N. Cartier et C. Butor, Paris, Flammarion Médecine Sciences, XIIII + 1294 p. [trad. de la $3^{\mathrm{e}}$ éd. de Molecular Biology of the Cell].

Morange, M. (1994): Histoire de la biologie moléculaire, Paris, Éditions de la Découverte, 357 p.

Lodish, H. et al., ed. (2000): Molecular Cell Biology, 4th ed., New York, Freeman \& Co., 1084 p + $\mathrm{G} 17+\mathrm{I} 36$.

Alberts, B. et al. (1998): Essential Cell Biology. An Introduction to the Molecular Biology of the Cell, New York, Garland Publishing, 630 p. + G18 + A62 + I30.

Jameson, J. L. et al., ed. (1998): Principles of Molecular Medicine, Totowa, Humana Press, xix + 1123 p.

MAUlik, S. (1997): Molecular Biotechnology: Therapeutic Applications and Strategies, New York and Toronto, Wiley-Liss, $\mathrm{xx}+223 \mathrm{p}$.

Elliotr, W. H. and D. C. (1997): Biochemistry and Molecular Biology, Oxford, Oxford University Press, $\mathrm{xxv}+437 \mathrm{p}$.

BeCKer, W. M. (1996): The World of the Cell, 3rd ed., Menlo Park, Benjamin / Cummings, xxiii + $897 \mathrm{p}$.

Alberts, B. et al. (1994): Molecular Biology of the Cell, 3rd ed., New York, Garland Publishing, xliii +1294 p. 


\subsection{Physiologie, physiopathologie et biochimie clinique}

BoREL, J.-P. (1999): Biochimie pour le clinicien: mécanismes moléculaires et chimiques à l'origine des maladies, Montréal, Maclean Hunter, 392 p.

Boulanger, P. et al. (1997): Biochimie médicale, $3^{e}$ éd., Paris, Masson, coll. "Abrégés ", 2 vol.

Boisseau, M. R. et al. (1996): Physiologie humaine, Paris, Pradel, 570 p.

Hennen, G. (1996): Biochimie humaine: introduction biochimique à la médecine interne, Bruxelles, De Boeck Université, 784 p.

MeYer, P. (1995): Physiologie humaine, $2^{e}$ éd., Paris, Flammarion Médecine Sciences, 1440 p.

Doré, D. (1994): Biochimie clinique, Sainte-Foy, Le Griffon d'argile; Paris, Maloine, 878 p.

SHeRwood, L. (1997): Human Physiology: from Cells to Systems, 3rd ed., Belmont, Wadsworth Publishing Co., xix +753 p.

Devlin, T. M., ed. (1997): Textbook of Biochemistry: with Clinical Correlations, 4th ed., New York and Toronto, Wiley, xxvii $+1186 \mathrm{p}$.

Gurton, A. C. et J. E. Hall (1997): Human Physiology and Mechanisms of Disease, Philadelphia and Montreal, Saunders, $x+737 \mathrm{p}$.

Montgomery, R. (1996): Biochemistry: A Case-Oriented Approach, 6th ed., Saint Louis and Toronto, Mosby, xvii $+683 \mathrm{p}$.

\subsection{Anatomie}

Kamina, P. (2000): Anatomie. Introduction à la clinique, Paris, Maloine, 12 vol.

- (2000): Dictionnaire atlas d'anatomie, Paris, Maloine, 3 vol.

- (1999): Petit atlas d'anatomie, Paris, Maloine, 170 p.

DAlley, A. F. (1999): Atlas d'anatomie humaine de F. H. Netter, $2^{\mathrm{e}}$ éd., trad. fr. P. Kamina, Paris, Maloine, $525 \mathrm{p}$.

Federative Committee on Anatomical Terminology (1998): Terminologia Anatomica, International Anatomical Terminology, Stuttgart and New York, Thieme.

DAlley, A. F. (1997): Atlas of human anatomy by F. H. Netter, 2nd ed., East Hanover (N.J.), Navartis, 525 p.

\subsection{Médecine interne}

Bennett, J.-C. et F. Plum, dir. (1997): Cecil-Traité de médecine interne, $1^{\text {re }}$ éd., Paris, Flammarion Médecine Sciences, xxxix +2339 p. [trad. de la $20^{\circ}$ éd. américaine]

Godeau, P. et al. (1996): Traité de médecine, $3^{\mathrm{e}}$ éd., Paris, Flammarion Médecine Sciences, xxxviI +2968 p.

Isselbacher, K. J., dir. (1995): Médecine interne. Harrison, $6^{\mathrm{c}}$ éd., Milan, Toronto et Paris, McGraw-Hill, 2 vol. [trad. de la $13^{e}$ éd. américaine].

Goldman, L., J. C. Bennett et al., ed. (2000): Cecil Textbook of Medicine, 21st ed., Philadelphia and Montreal, Saunders, $\mathrm{XLI}+2308 \mathrm{p}$.

FAUcI, A. S. et al., ed. (1998): Harrison's Principles of Internal Medicine, 13th ed., New York and Montreal, McGraw-Hill, xxvi $+2569+170$ p. [disponible sur Internet par abonnement: $<$ http://www.harrisonline.com $>$ )

\subsection{Cardiologie}

Cohen, A. (1997): Cardiologie et pathologie vasculaire, Paris, Estem, 1113 p.

Topol, E. J. and R. M. Califf, ed. (1998): Comprehensive Cardiovascular Medicine, Philadelphia, Lippincott-Raven, xxix +3153 p.

Willerson, J. T. et J. N. Cohn, ed. (1995): Cardiovascular Medicine, New York, Churchill Livingston, xvi $+1976 \mathrm{p}$. 


\subsection{Immunologie}

BACH, J.-F. (1999): Immunologie, $3^{e}$ éd., Paris, Flammarion Médecine Sciences, coll. "De la biologie à la clinique ", $420 \mathrm{p}$.

Revillard, J.-P. (1998): Immunologie, $3^{e}$ éd., Paris, De Boeck Université, 461 p.

Rortт, I. M. (1997): Immunologie, $4^{e}$ éd., Bruxelles, De Boeck Université, xii + 406 p. [trad. d'Immunology, $4^{\mathrm{e}}$ éd., par J.-P. Revillard et W. H. Fridman].

Gennetet, N., dir. (1997): Immunologie, Rennes et Cachan, Éditions Médicales Internationales, $604 \mathrm{p}$.

Cruse, J. M. (1999): Atlas of Immunology, Boca Raton, CRC Press, 451 p.

PAUL, W. E., dir. (1999): Fundamental Immunology, Philadelphia and New York, LippincottRaven, xviii + 1589 p.

Roitt, I., J. Brostoff and D. MAle (1998): Immunology, 5th ed., London, Mosby, viii + 423 p.

Kuby, J. (1997): Immunology, 3rd ed., New York, W. H. Freeman, xxix + 664 p.

Stites, D. P., A. I. Terr and T. G. PArslow, ed. (1997): Medical Immunology, Stamford, Appleton \& Lange, $x i i+900$ p.

\subsection{Génétique médicale}

Kaplan, J.-C. et M. Delpech (1998) : Biologie moléculaire et médecine, $2^{e}$ éd., Paris, Flammarion Médecine Sciences, 750 p.

Rossignol, J.-L. (1996): Génétique, Paris, Masson, xii + 477 P.

Thompson, M. W. et al. (1995): Génétique médicale, Paris, Flammarion Médecine Sciences, coll. "De la biologie à la clinique", xii +495 p. [trad. de Genetics in medicine, $5^{e}$ éd., par M. Vekemans].

Wilson, G. N. (2000): Clinical Genetics. A Short Course, New York, Wiley-Liss, xii +477 p.

Jameson, J. L. (1998): Principles of Molecular Medicine, Totowa, Humana Press, xix +1123 p.

Ross, D. W. (1996): Introduction to Molecular Medicine, 2nd ed., New York, Springer, xii +178 p.

Tномpson, M. W. et al. (1991): Thompson \& Thompson Genetics in Medicine, 5th ed., Philadelphia, W. B. Saunders, $x i+500$ p.

\subsection{Neurosciences et neurologie}

Bear, M. F. (1999): Neurosciences. À la découverte du cerveau, Paris, Pradel, 654 p. [trad. et adapt. de Neuroscience: Exploring the brain par A. Nicoullon].

Cambier, J. et al. (1998): Neurologie, $9^{c}$ éd., Paris, Masson, xiii + 595 p.

Serratrice, G. et al. (1996) : Neurologie, Paris, Ellipses, 704 p.

Cousin, F.-R. et G. Dubret (1989): Eléments d'anatomie et de physiologie du système nerveux central, Paris, Flammarion Médecine Sciences, coll. "Monographies », 416 p.

Kandel, E. et al., ed. (2000): Principles of Neural Sciences, 4th ed., New York, Elsevier, xli + 1414 p. Gilroy, J. (2000): Basic Neurology, 3rd ed., New York and Montreal, McGraw-Hill, viii + 702 p. Martin, J. H. (1996): Neuroanatomy. Text and Atlas, 2nd ed., New York, McGraw Hill, xviii + $578 \mathrm{p}$.

\subsection{Oncologie}

Daly-Schveitzer, N. (1998): Cancérologie clinique, Paris, Masson, xxi + $461 \mathrm{p}$.

DeVita, V. T. et al., ed. (1997): Cancer: Principles \& Practice of Oncology, Philadelphia, J. B. Lippincott, lxviii + 3125 p.

\subsection{Constantes biologiques et analyses médicales}

DURAND, H. et P. Biclet (1999): Dictionnaire des examens biologiques et investigations complémentaires, Paris, Doin, 836 p. [existe aussi en cédérom]. 
HARLey, A. (1997): Dictionnaire des examens complémentaires en médecine, Paris, Masson, xiv + $305 \mathrm{p}$.

Dieusaert, P. (1996): Guide pratique des analyses médicales, Paris, Maloine, vii +1223 p.

Blaque-BélaIr, A. et al. (1991): Dictionnaire des constantes biologiques et physiques en médecine. Applications cliniques pratiques, $6^{\mathrm{e}}$ éd., Paris, Maloine, $842 \mathrm{p}$.

Pagana, K. D. and T. J. Pagana (1997): Mosby's Diagnostic and Laboratory Test Reference, 3rd ed., St. Louis and Toronto, Mosby, xxv + $944 \mathrm{p}$.

Tietz, N. W., ed. (1995): Clinical Guide to Laboratory Tests, 3rd ed., Philadelphia and Montreal, Saunders, xxxix +1096 p.

\subsection{Sciences pharmaceutiques}

Decodts, G. (1997): Les bases de la chimie organique. Cours et exercices corrigés, $2^{e}$ éd., Paris, Flammarion Médecine Sciences, $244 \mathrm{p}$.

LE Hir, A. (1997): Pharmacie galénique: bonnes pratiques de fabrication des médicaments, $7^{\mathrm{C}}$ éd., Paris, Masson, coll. "Abrégés de pharmacie», xvii + 381 p.

HazebroucQ, G., dir. (1995): Dorvault. L'officine, $23^{\mathrm{e}}$ éd., Paris, Vigot, xxvii + 2089 p.

Fattorusso, V. (1992): Dictionnaire des médicaments avec répertoire des dénominations communes internationales d'après l'OMS, Paris, Masson, vi + 992 p.

Boudet-Dalbin, R. (1992): Dénomination chimique. Traité de chimie thérapeutique, vol. 1, Paris, Techniques \& Documentation; Lavoisier, Éditions Médicales et Internationales, 373 p.

AIACHE, J.-M. et al. (1989): Initiation à la connaissance du médicament, Paris, Masson, coll. "Abrégés de pharmacie", $x v+268$ p.

Wu-Pong, S. et Y. Rojanasakul (1999): Biopharmaceutical Drug Design and Development, Totowa, Humana Press, xii +435 p.

Gennaro, A. R. (1995): Remington: the Science and Practice of Pharmacy, Easton, Mack Publications, xvi +1934 p. [existe aussi en cédérom].

\subsection{Répertoires de médicaments}

5.13.1. Etats-Unis

Complete drug reference (2000), United States Pharmacopeia, New York, Consumer Reports Books, 1760 p. [mises à jour annuelles]

Drug Information for the Health Professional (2000), 20th ed., United States Pharmacopeia, New York and Englewood, Micromedex, xxvi +3548 p. [mises à jour annuelles].

5.13.2. Canada

Compendium des produits et spécialités pharmaceutiques / Compendium of Pharmaceuticals and Specialties (annuel), Ottawa, Association des pharmaciens du Canada / Canadian Pharmacists Association.

Compendium of Nonprescription Products, Ottawa, Association des pharmaciens du Canada / Canadian Pharmacists Association.

5.13.3. France

Le dictionnaire Vidal (annuel), Paris, OVP-Éditions du Vidal [trois mises à jour par an].

\subsection{Pharmacologie}

Hardman, J. G. et al., dir. (1998): Les bases pharmacologiques de l'utilisation des médicaments, Londres et Montréal, McGraw-Hill International, xxvi + 1677 [258] p. [trad. de Goodman \& Gilman's The Pharmacological Basis of Therapeutics, $9^{\mathrm{e}}$ éd.; revue scientifique de l'édition française: Tillement, Jean-Paul et al.].

SCHORDERET, M., dir. (1998): Pharmacologie: des concepts fondamentaux aux applications thérapeutiques, $3^{\mathrm{e}}$ éd., Paris, Frison-Roche; Genève, Slatkine, xiii +1010 p. 


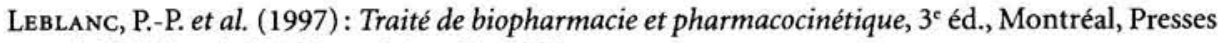
de l'Université de Montréal, xxiii + 396 p.

Giroux, J.-P. et al., dir. (1988): Pharmacologie clinique. Bases de la thérapeutique, $2^{e}$ éd., Paris, Expression Scientifique Française, xxiii +2352 p.

Hardman, J. G. et al., ed. (1996): Goodman \& Gilman's The Pharmacological Basis of Therapeutics, 9th ed., New York and Montreal, McGraw-Hill, xxi + 1905 p.

\subsection{Essais cliniques et épidémiologie}

5.15.1. Monographies publiées en France

Bouvenot, G. et M. VRaY (1996): Essais cliniques: théorie, pratique et critique, $2^{\mathrm{c}}$ éd., Paris, Flammarion Médecine Sciences, xxi $+409 \mathrm{p}$.

Landrivon, G. et F. Delahaye, dir. (1995): La recherche clinique. De l'idée à la publication, Paris, Masson, xviii $+273 \mathrm{p}$.

Bouyer, J. et al. (1994) : Épidémiologie. Principes et méthodes quantitatives, Paris, INSERM, 209 p.

Caulin, C. et al., dir. (1993): Méthodologie de l'évaluation thérapeutique, Paris, Masson, $\mathrm{x}+340 \mathrm{p}$.

SPRIET, A. et al. (1993): Méthodologie des essais cliniques des médicaments, $3^{ }$éd., Paris, Karger, viii +269 p.

\subsubsection{Monographies publiées au Québec}

Beaucage, C. et Y. Bonnier-VIger, dir. (1996): Épidémiologie appliquée: une initiation à la lecture critique de la littérature en sciences de la santé, Montréal, G. Morin, xxv $+550 \mathrm{p}$.

Jenicex, M. et R. Cléroux (1993): Épidémiologie. Principes, techniques, applications, SaintHyacinthe, Edisem; Paris, Maloine, $454 \mathrm{p}$.

Jenicek, M. (1987): Méta-analyse en médecine: évaluation et synthèse de l'information clinique et épidémiologique, Saint-Hyacinthe, Edisem; Paris, Maloine, 152 p.

JeNICEK, M. (1995): Epidemiology: the Logic of Modern Medicine, Montreal, Epimed, xi + 335 p.

5.15.3. Monographies publiées aux États-Unis

Rothman, K. J. and S. Greenland (1998): Modern Epidemiology, 2nd ed., Philadelphia, Lippincott-Raven, xiii $+738 \mathrm{p}$.

Kelsey, J. L. et al. (1996): Methods in Observational Epidemiology, 2nd ed., New York, Oxford University Press, viii $+432 \mathrm{p}$.

PAK, C. Y. C. et P. A. AdAms (1994): Techniques of Patient-Oriented Research, New York, Raven Press, $\mathrm{xi}+205 \mathrm{p}$.

SPILKer, B. (1991): Guide to Clinical Trials, New York, Raven Press, xxv + 1156 p.

\subsection{Statistiques biomédicales}

Falissard, B. (1996): Comprendre et utiliser les statistiques dans les sciences de la vie, Paris, Masson, coll. "Evaluation et statistique», xii + 314 p.

BOUYER, J. et al. (1995) : Épidémiologie: principes et méthodes quantitatives, Paris, INSERM, xiii + $498 \mathrm{p}$.

Esteve, J., E. Benhamou et L. Raymond (1993): Méthodes statistiques en épidémiologie descriptive, Paris, INSERM, xiii $+307 \mathrm{p}$.

SAhat, H. et A. KhURshid (1996) : Statistics in Epidemiology: Methods, Techniques, and Applications, Boca Raton, CRC Press, 321 p.

Selvin, S. (1996): Statistical Analysis of Epidemiologic Data, 2nd ed., New York, Oxford University Press, xvii $+467 \mathrm{p}$.

Page, R. M., G. E. Cole and T. C. Trmmreck (1995): Basic Epidemiological Methods and Biostatistics: a Practical Guidebook, Boston, Jones and Bartlett, xiii +433 p.

Анцвом, А. (1993): Biostatistics for Epidemiologists, Boca Raton, Lewis Publishers, ix + 214 p. 
Clayton, D. (1993): Statistical Models in Epidemiology, Oxford and New York, Oxford University Press, viii +367 p.

\subsection{Pharmacoéconomie et économie de la santé}

Crochard-Lacour, A. et J. LeLorier (2000): Introduction à la pharmacoéconomie, Montréal, Presses de l'Université de Montréal, $93 \mathrm{p}$.

GRENIER, B. (1996) : Évaluation de la décision médicale: introduction à l'analyse médico-économique, Paris, Masson, coll. "Évaluation et statistique ", xiv + 412 p.

Moatтı, J.-P., dir. (1996): Recherche clinique et qualité de vie, Paris, Flammarion Médecine Sciences, $\mathrm{vi}+184 \mathrm{p}$.

Beresniak, A. et al. (1995): Économie de la santé, $3^{\mathrm{e}}$ éd., Paris, Masson, viii $+182 \mathrm{p}$.

SPILKer, B. (1996): Quality of Life and Pharmacoeconomics in Clinical Trials, Philadelphia, Lippincott-Raven, xiv + 1259 p.

\subsection{Abréviations et éponymes}

VAN Hoof, H. (1999): Dictionnaire des abréviations, acronymes et symboles utilisés en médecine: anglais/français = Dictionary of Abbreviations, Acronyms and Symbols Used in Medicine: French/English, Paris, La Maison du dictionnaire, $\mathrm{xv}+221 \mathrm{p}$.

Dionne, S. et J. Boulay (1998): Guide bilingue des abréviations médicales, $2^{\mathrm{c}}$ éd., Montréal, Maclean Hunter Santé, $73+81$ p.

MAIllet, M. (1996): Dictionnaire des abréviations en biologie: 2500 abréviations et acronymes, Paris, Masson, vi +237 p.

VAN Hoof, H. (1993): Dictionnaire des éponymes médicaux français-anglais, Louvain-la-Neuve, Peeters, 405 p.

— (1986): «Les éponymes médicaux: essais de classification», Meta, 31-1, p. 59-84.

Davis, N. M. (1999): Medical Abbreviations, 9th ed., Huntington Valley, Neil M. Davis Associates, 380 p. [voir <http://www.neilmdavis.com>].

SLoAne, S. B. (1997): Medical Abbreviations \& Eponyms, 2nd ed., Philadelphia and Montreal, Saunders, $x+905 p$.

JABLONSKI, S. (1991): Jablonski's Dictionary of Syndromes and Eponymic Diseases, 2nd ed., Malabar, Krieger Publishing Co., ix +665 p.

\section{Articles traitant de langue ou de traduction médicales}

Fischвach, H., ed. (1998): Translation and Medicine, Amsterdam and Philadelphia, John Benjamins Publishing, coll. "American Translators Association Scholarly Monograph Series", vol. 10, $194 \mathrm{p}$.

- (1993): "Translation, the Great Pollinator of Science: a Brief Flashback on Medical Translation," Scientific and Technical Translation (S. E. WrIGHT, ed.), Amsterdam, John Benjamins Publishing, pp. 89-100.

- (1986): "Some Anatomical and Physiological Aspects of Medical Translation Lexical Equivalence, Ubiquitous References and Universality of Subject Minimize Misunderstanding and Maximize Transfer of Meaning," Meta, 31-1, p. 16-21.

GABRIELli, E. R. (1986) : “Construction of a Biomedical Nomenclature," Meta, 31-1, p. 22-25.

JAMMAL, A. (1999) : «Une méthodologie de la traduction médicale », Meta, 44-2, p. 217-237.

Soubrier, J. (1998) : "Problèmes de traduction dans le domaine de la chirurgie orthopédique", Traducción e interpretación en el ambito biosanitario, Actos del Seminario internacional de traducción e interpretación en el ambito biosanitario, Universidad de Malaga (30 mars au 2 avril 1998), Malaga, Comares, p. 251-267.

Rouleau, M. (1995): compte rendu du Dictionnaire des éponymes médicaux français-anglais, Meta, 40-4, p. 680-681. 
- (1995): "La langue médicale: une langue de spécialité à emprunter le temps d'une traduction ", TTR, 8, p. 29-49.

Sournia, J.-C. (1994): "Les phases évolutives du vocabulaire médical français", Meta, 39-4, p. 692-700.

JammaL, A. et al. (1994): "L'immunologie et son vocabulaire français", L'actualité terminologique, 27-3, p. 15-21; 28-4, p. 16-24.

Rouleau, M. (1993): «La voix passive dans les textes médicaux et paramédicaux», Meta, 38-3, p. $440-448$.

- (1993): "Des traquenards de la version médicale: action, effect, potency and effectiveness ", Meta, 38-2, p. 268-274.

Jammal, A. et al. (1993) : "Si l'immunologie m’était contée... ", Meta, 38-3, p. 502-517.

Rouleau, M. (1992): Compte rendu de trois vocabulaires du Secrétariat d'État, Meta, 37-2, p. 360367.

JAMMAL, A. (1992): "L'hybridation du langage médical français: un phénomène inévitable ", J. Radiol., 73, p. 213-214.

VAliquette, M. (1991) : «Lexique anglais-français de neurochirurgie», Meta, 36-4, p. 633-644.

Rouleau, M. (1991): "Adverse effect est-il synonyme de side-effect?", Meta, 36-2/3, p. 497-507.

JAMmaL, A. (1990): "L'étude des langues des spécialités médicales, un scyalitique sur un champ opératoire", Meta, 35-1, p. 50-54.

- (1989): "Étiologie de certains brouillages terminologiques de la langue médicale », Meta, 34-4, p. 764-769.

- (1988): "Les vocabulaires des spécialités médicales: comment et pourquoi les fabrique-ton? ", Meta, 33-4, p. 535-541.

Bélanger, L. (1986): "Bibliographie / Bibliography. Sciences médicales / Medical Sciences", Meta, 31-1, p. 106-118.

MOREAU, A. (1986) : "La traduction médicale: réflexions de praticiens. Enquête d'André Moreau ", Meta, 31-1, p. 98-105.

Martin-Valiquette, L. (1986): "Les traquenards de la traduction médicale... ou l'interaction texte-traducteur-dictionnaire ", Meta, 31-1, p. 31-33.

GILE, D. (1986) : «La traduction médicale doit-elle être réservée aux seuls traducteurs-médecins? Quelques réflexions ", Meta, 31-1, p. 26-30.

Sournia, J.-C. (1986): "Les dictionnaires médicaux vus par un médecin ", Meta, 31-1, p. 7-10.

Jammal, A. et al. (1986): "L'épidémiologie et les mots pour le dire ", Meta, 31-1, p. 34-58.

- (1983): "L'hypertension artérielle et les singularités de son vocabulaire ", Meta, 28-4, p. 376390.

- (1981): «Les problèmes terminologiques de l'allergie ", Meta, 26-4, p. 378-381.

- (1980): "La terminologie, pour quoi faire?", Meta, 25-4, p. 467-469.

VAN Hoof, H. (1974) : « Bibliographie de la traduction médicale ", Meta, 19-1, p. 43-66.

Eyraud, D. (1974): "Bilan d'une décennie ", Meta, 19-1, p. 13-27.

Manuila, A. et A. Rigolot (1974): «Le français, langue médicale internationale ", Meta, 19-1, p. 3-12.

Pharmaterm, bulletin terminologique du Groupe traduction, Les compagnies de recherche pharmaceutique du Canada².

\section{NOTES}

1. La toute dernière nomenclature internationale, sortie en 1998, s'intitule Terminologia anatomica et comporte des équivalents anglais et latins. Elle remplace la Nomina anatomica publiée en 1989. Elle devrait servir de base à l'harmonisation de la terminologie pour tous les pays (voir Whitmore 1999 pour une présentation de cette nouvelle nomenclature et un historique des précédentes). Il est important de vérifier la nomenclature utilisée lorsqu'on consulte un ouvrage d'anatomie. (Ian Whitmore (1999): "Terminologia Anatomica: New Terminology for the New Anatomist," Anatomical Record, 257-2, pp. 50-53.) 
2. Pour s'abonner à Pharmaterm, contacter $\mathrm{M}^{\text {m:e }}$ Marina de Almeida, Wyeth-Ayerst Canada Inc., 1025 boul. Marcel-Laurin, Saint-Laurent (Québec), H4R 1J6, Canada.

\section{REMERCIEMENTS}

Le présent travail a pu être réalisé grâce à des subventions de l'Université de Montréal. L'auteure remercie Maurice Rouleau et Serge Quérin pour leurs suggestions, ainsi que Micheline Roberge et Manon Pageau pour leur contribution à la collecte des documents et des données. 